\title{
Geometric phases of scattering states in a ring geometry: adiabatic pumping in mesoscopic devices
}

\author{
Huan-Qiang Zhou† §, Urban Lundin†, and Sam Young Cho $\dagger$ \\ † School of Physical Sciences, University of Queensland, Brisbane Qld 4072, Australia
}

\begin{abstract}
Geometric phases of scattering states in a ring geometry are studied based on a variant of the adiabatic theorem. Three time scales, i.e., the adiabatic period, the system time and the dwell time, associated with adiabatic scattering in a ring geometry plays a crucial role in determining geometric phases, in contrast to only two time scales, i.e., the adiabatic period and the dwell time, in an open system. We derive a formula connecting the gauge invariant geometric phases acquired by time-reversed scattering states and the circulating (pumping) current. A numerical calculation shows that the effect of the geometric phases is observable in a nanoscale electronic device.
\end{abstract}

PACS numbers: 03.65.Vf, 03.65.Nk, 73.23.-b

Submitted to: J. Phys.: Condens. Matter

$\S$ To whom correspondence should be addressed (hqz@maths.uq.edu.au) 


\section{Introduction}

The study of geometric phases continues to be an intriguing subject. Since Berry's original work [1], various generalizations have been proposed [2, 3] to adapt to different physical applications in diverse fields. In Ref. [4] the concept of geometric phases for scattering states was introduced which allows to give a geometric interpretation of the formalism developed by Brouwer [5] for quantum adiabatic pumping in open systems. Specifically, Brouwer presented a compact formula for the pumped charge (current) in terms of the parametric derivatives of the time-dependent scattering matrix subjected to the oscillating potential, which has been identified as the geometric phase accompanying the scattering state associated with the time-reversed Hamiltonian. Quantum adiabatic pumping in open systems is subject to intense study $[5,6,4,7]$ due to the observation of adiabatic electron transport through a quantum dot subject to slow cyclic variation of gate voltages [8].

An intriguing question has been raised recently about the possibility to generate a circulating (pumping) current in a ring geometry by adiabatically varying external parameters [9, 10]. In Ref. [9], Cohen considered open systems as some subtle limit of closed systems and demonstrated that it is possible to reproduce the formulas by Landauer [11] and Büttiker, Prètre and Thomas [12] from the Kubo formula whereas Moskalets and Büttiker [10] examined this problem in the slow frequency limit of the Floquet scattering theory. These differences in approaches mean that the results are different, although they aimed at describing the same system. Such an unsatisfying situation calls for a thorough analysis of the problem. In this Letter, we address this issue from gauge field and geometric phase perspectives. We derive a compact formula connecting the circulating (pumping) current [13] and the gauge invariant geometric phases acquired by time-reversed scattering states. This is achieved by a purely geometric argument, based on the fact that pumped charge is additive for two consecutive pumping cycles. A generic feature of pumping in a ring geometry is that the momentum is time-dependent and so are the discrete energy levels. The closed system is different from the open in another aspect. In an open system there are only two different time scales, whereas in the closed ring geometry three different time scales appear, the adiabatic period, the system time and the dwell time. The physics behind this is the following. In the adiabatic limit, electrons are in an instantaneous scattering state. A small deformation in the scattering potential results in a small increment of geometric

phases, which electrons can pick up at the dwell time scale. The dwell time being the time it takes for an electron to complete one scattering event. Since the electrons travel in a ring geometry, they have to obey the periodic boundary condition to which they adjust at the system time scale, $\tau_{s}$. All electronic wave functions are extended over the entire ring thus inducing a circulating current. The latter is experimentally measurable in terms of a SQUID that measures the magnetic field produced by the circulating current. 


\section{The system}

Consider a quantum dot with two leads attached such that the two single-channel leads are bent back to form a ring [see Fig. 1(a)]. Suppose the dot is characterized

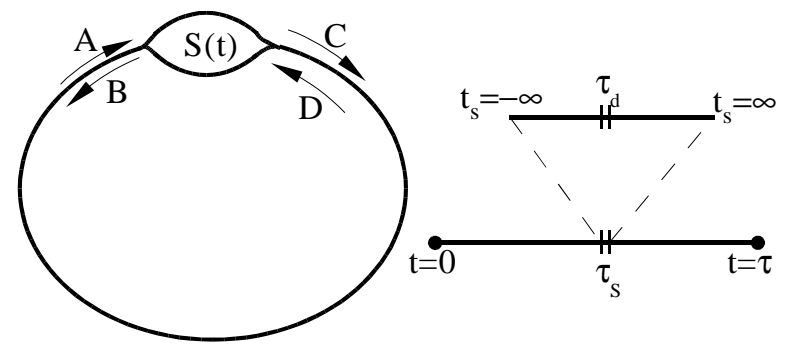

(a)

(b)

Figure 1. (a) shows the setup with a time dependent scatterer, defined by its scattering matrix, $S(t)$. Incident, $A$, and reflected wave, $B$. (b) shows the time scales relevant to the problem. Scattering takes place on $\tau_{s}$, the dwell time (the time it takes for the scattering event) is $\tau_{d} . t_{s}$ designates the time passing for the scattering event, it is only valid on the $\tau_{s}$-scale. The time it takes for the wave function to adjust the boundary conditions to the scatterer, of the order of $\tau_{s}$, must be shorter than the adiabatic changes in the scatterer, happening on the scale $\tau$.

by a scattering matrix $S(t)$ which depends on time $t$ via a set of independent external parameters $X \equiv\left(X^{1}, \cdots, X^{\nu}, \cdots, X^{p}\right)$ oscillating slowly with frequency $\omega$. For a spinindependent scatterer, $S(t)$ is a $2 \times 2$ matrix. Define the row vectors $\mathbf{n}^{L} \equiv(r, t)$, and $\mathbf{n}^{R} \equiv\left(t^{\prime}, r^{\prime}\right)$, the unitarity of the scattering matrix implies that they are orthonormal $\mathbf{n}^{\alpha} \cdot \mathbf{n}^{\beta}=\delta_{\alpha \beta}$, where $\alpha, \beta=L, R$. From the boundary condition of the matching of the wavefunction for a ring geometry, the dispersion equation is written in terms of scattering matrices, $\operatorname{det}\left(S S_{w}-1\right)=0$ with $S_{w}=\exp (i k L) \sigma^{x}$, where $\sigma^{x}$ is the Pauli matrix. $S_{w}$ is the scattering matrix describing the remaining part of the ring except the dot. The energy spectrum is then discrete in the ring geometry. The adiabaticity requires that the frequency $\omega$ is small enough compared with the (averaged) level spacing $\Delta$. On the other hand, for the instantaneous scattering matrix to make sense, it is necessary that the system time $\tau_{s} \equiv L / v$ ( $v$ - electron velocity) is much greater than the dwell time $\tau_{d}[14]$ during which electrons scatter off the scatterer. That is, in the ring geometry, there are three different time scales: the period $\tau=2 \pi / \omega$, the system time $\tau_{s}=\hbar \Delta^{-1}$, and the dwell time, $\tau_{d}$. In the scattering approach to adiabatic quantum pumping in the ring geometry, we assume $\tau>>\tau_{s}>>\tau_{d}$. This condition ensures that it is legitimate to speak of instantaneous scattering states, consistent with Heisenberg uncertainty principle [15]. We illustrate this in Fig. 1(b) and notice that it is different from the adiabaticity condition discussed in Ref. [10]. Generically, the allowed (discrete) $k$ 's, which in turn determine the discrete energy levels, are time-dependent due to the fact that the external parameters defining the scatterer $X \equiv\left(X^{1}, \cdots, X^{\nu}, \cdots, X^{p}\right)$ are changing with time and the scattering matrix are decisive for the dispersion, a feature different from scattering in open systems. 
Discretized approach and the continuous limit. Suppose the system undergoes an adiabatic cycle with the period $\tau$, which is characterized by a closed loop in parameter space. Suppose that initially an electron is in a given discrete energy level, the adiabaticity ensures that it remains in the same level during the entire period. To take into account the time scales properly, we adopt a discretized approach, i.e., the entire interval $[0, \tau]$ is divided into $N$ pieces. Correspondingly, the closed loop may be regarded approximately as a polygon [see Fig. 2(a)]. Here we stress that each subinterval

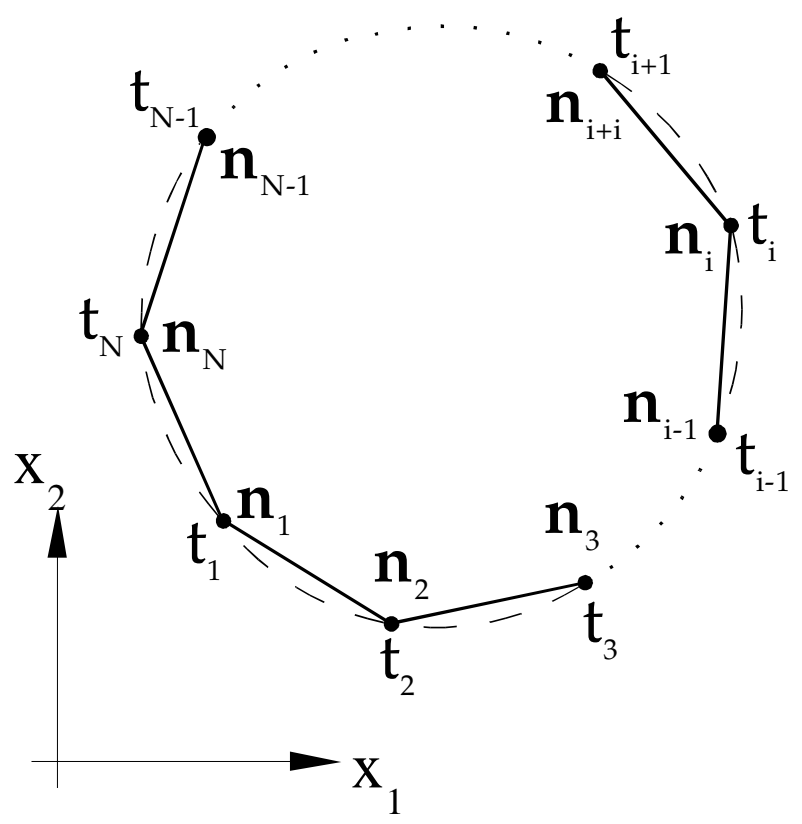

(a)

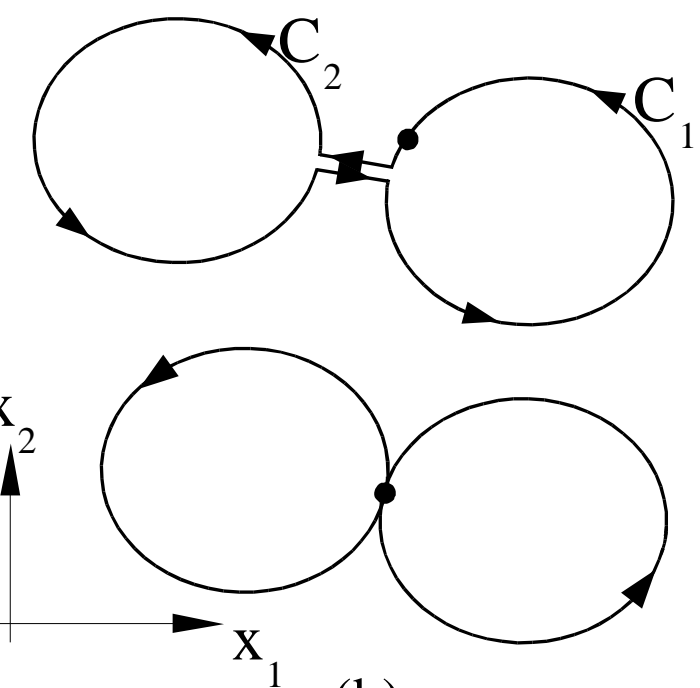

(b)

Figure 2. (a) displays the discretization of the adiabatic loop. Doing this, we can ensure that the scattering matrix formalism gives a correct description and distinguish the time scales associated with the theory. Here, the entire interval $[0, \tau]$ is divided into $N$ pieces. Each subinterval $\left[t_{i}, t_{i+1}\right]$ is of the same order of magnitude as the system time $\tau_{s}$. At each instant, $t_{i}$, two new vectors $n_{i}^{L}$ and $n_{i}^{R}$ are defined in terms of the instantaneous $S$-matrix. (b) displays the property that the geometric phase is additive, and the pumped charge being a function of the geometric phase has to be additive. The lower contour displays a special case in which two cycles share the same initial configuration, represented by a dot, whereas the upper contour shows two separate cycles joined by two adjacent but opposite lines.

$\left[t_{i}, t_{i+1}\right]$ is at the same order of magnitude as the system time $\tau_{s}$. At each instant $t_{i}$, we have two row vectors $\mathbf{n}_{i}^{L}$ and $\mathbf{n}_{i}^{R}$. Since the argument is parallel, we drop the superscripts $L$ and $R$ for brevity. For two consecutive instants $t_{i}$ and $t_{i+1}$, we may define the relative phase between the two vectors $\mathbf{n}_{i}$ and $\mathbf{n}_{i+1}$ by $\exp \left(i \phi_{i}\right)=\mathbf{n}_{i}{ }^{*} \cdot \mathbf{n}_{i+1} /\left|\mathbf{n}_{i} \cdot \mathbf{n}_{i+1}^{*}\right|$. That is, $\phi_{i}=\operatorname{Im} \ln \mathbf{n}_{i}{ }^{*} \cdot \mathbf{n}_{i+1}$. Therefore we have the total phase for the closed loop (polygon)

$$
\phi \equiv \sum_{i=1}^{N} \phi_{i}=\operatorname{Im} \ln \prod_{i=1}^{N} \mathbf{n}_{\mathbf{i}}{ }^{*} \cdot \mathbf{n}_{i+1} .
$$


That is, both $\mathbf{n}^{L}$ and $\mathbf{n}^{R}$ acquire a geometric phase $\phi^{L}$ and $\phi^{R}$, respective, if the system returns to the initial configuration after one adiabatic cycle. Indeed, Eq. (1) is invariant under the (local) gauge transformation $\mathbf{n}_{i}^{\prime}=e^{i \alpha_{i}} \mathbf{n}_{i}$, which arises from the fact that the absolute phase is not observable in quantum mechanics. In the continuum limit, we have

$$
\phi=\operatorname{Im} \oint \mathbf{n}^{*} \cdot d \mathbf{n} .
$$

This amounts to the statement that the gauge potential $A$ is $\operatorname{Im}\left(\mathbf{n}^{*} \cdot d \mathbf{n}\right)$ up to an (integrable) immaterial term. Indeed, in the adiabatic limit, it is reasonable to assume that the gauge potential $A$ only depends on $\mathbf{n}, d \mathbf{n}, k, d k$ and $L$. From this we see that the most general gauge potential we can construct takes the form $A=\mathbf{n}^{*} \cdot d \mathbf{n}+g(k L) d k L$ with $g$ an arbitrary function, if we require that $A$ transforms as

$$
A^{\prime}=i d \alpha+A
$$

under the gauge transformation. Here the second term proportional to $d k L$ comes from the dependence of discrete $k$ 's on time. Below we will see that $g=i / 4$. Although the gauge potential $A$ is formally identical to its counterpart in the case of open systems when expressed in terms of the row vector $\mathbf{n}$, the explicit dependence of $\mathbf{n}$ on $k$ which varies with time characterizes the difference.

Similarly, we may define two column vectors $\hat{\mathbf{n}}^{L} \equiv\left(r, t^{\prime}\right)$, and $\hat{\mathbf{n}}^{R} \equiv\left(t, r^{\prime}\right)$, which are orthonormal due to the unitarity of the $S$ matrix. Repeating the above argument, we conclude that $\hat{\mathbf{n}}^{L}$ and $\hat{\mathbf{n}}^{R}$ acquire, respectively, geometric phases $\hat{\phi}^{L}$ and $\hat{\phi}^{R}$, which take the form, $\hat{\phi}=\operatorname{Im} \oint \hat{\mathbf{n}}^{*} \cdot d \hat{\mathbf{n}}$. Here the hat denotes the time-reversal operation since the row vectors and column vectors are connected via time reversal operation.

Consistency with the periodic boundary condition. The above discussion indicates that there is a gauge group $U_{L}(1) \times U_{R}(1)$, which is necessary to accommodate the geometric phases $\phi_{L}$ and $\phi_{R}$ acquired by the two row vectors $\mathbf{n}_{L}$ and $\mathbf{n}_{R}$, respectively. As we see, this results from the gauge freedom associated with the incident waves. In contrast to adiabatic pumping in open systems, one has to keep in mind that the scattering wave functions should satisfy the periodic boundary condition in the ring geometry. If we set $\Psi_{L}(x)=A \exp (i k x)+B \exp (-i k x)$ for $-L / 2 \leq x \leq 0$ and $\Psi_{R}(x)=C(i k x)+D \exp (-i k x)$ for $0 \leq x \leq L / 2$, then the incident waves and the scattered waves are connected via $B=A r+D t^{\prime}$ and $C=A t+D r^{\prime}$. On the other hand, the period boundary condition requires that $B=D \exp (-i k L)$, and $C=A \exp (-i k L)$. Besides the dispersion equation which results in discrete energy spectrum, we have $D=A r /\left(\exp (-i k L)-t^{\prime}\right)$. Formally, we write the scattering state $\psi$ as $\psi=A \psi_{L}+D \psi_{R}$ with $\psi_{L}=\exp (i k x)+r \exp (-i k x)+t \exp (i k x)$ and $\psi_{R}=$ $\exp (-i k x)+r^{\prime} \exp (i k x)+t^{\prime} \exp (-i k x)$. Then the $U_{L}(1) \times U_{R}(1)$ gauge transformation takes the form $\psi_{L(R)} \rightarrow \exp \left(i \alpha_{L(R)}\right) \psi_{L(R)}$, which induces transformations on $A$ and $D: A \rightarrow \exp \left(-i \alpha_{L}\right) A$ and $D \rightarrow \exp \left(-i \alpha_{R}\right) D$. Then we see that under such a gauge transformation the dispersion equation remains the same, but an extra phase factor $\exp \left(-i\left(\alpha_{L}-\alpha_{R}\right)\right)$ appears in the relation between $A$ and $D$. With $\psi_{L}$ and $\psi_{R}$ as the 
local basis, the time evolution governed by the Schrödinger equation induces parallel transport [16]. Taking into account the causality condition which states that scattered waves appear only after incident waves hit the scatterer, we may derive the expression of the gauge potential $A$ (where $g$ is determined to be i/4), resulting in the geometric phases $\phi_{L}$ and $\phi_{R}$. When the system is brought back to the initial configuration, the scattering states $\psi_{L}$ and $\psi_{R}$ acquire, respectively, geometric phases $\phi_{L}$ and $\phi_{R}$. Now we may picturize the observable effect of geometric phases as follows. Suppose the system undergoes an adiabatic cycle. During this process, at each instant, electrons are in an instantaneous scattering state. When electrons feel a slow deformation in the scattering potential, they pick up a small increment of geometric phases at the dwell time $\tau_{d}$ scale, and then take time at the system time $\tau_{s}$ scale to redistribute themselves to maintain the periodic boundary conditions, thus inducing the circulating current in the pumping experiment setup.

In the above we focused on scattering geometric phases acquired by scattering states. However, in the pumping setup, physical observables are connected with pumping geometric phases associated with time-reversed scattering states, a fact already known for pumping in open systems. In fact, there is another gauge group $U_{L}(1) \times U_{R}(1)$ associated with the gauge freedom of the scattered waves, which accommodates the geometric phases $\hat{\phi}_{L}$ and $\hat{\phi}_{R}$.

Connection between physical observables and geometric phases. Now let us establish the connection between the geometric phases $\hat{\phi}_{L}$ and $\hat{\phi}_{R}$ and the physical observables which are the charge $Q_{a}$ accumulated inside the dot and the charge $Q_{c}$ circulating in the ring during the entire pumping period $\tau$ for a given occupied level. Suppose $Q^{L}$ and $Q^{R}$ are, respective, the charges pumped from the dot to the left and right sides. Obviously, we have $Q_{a}=-\left(Q^{L}+Q^{R}\right)$. As for $Q_{c}$, one may expect that it is proportional to $\left(Q^{L}-Q^{R}\right)$, i.e., . $Q_{c}=\xi\left(Q^{L}-Q^{R}\right)$ with $\xi$ some undetermined constant. To determine $\xi$, we consider the special case in which no charge is accumulated inside the dot. In such a case, we have $Q^{L}=-Q^{R}=Q$. That is, the amount of charge $\mathrm{Q}$ is pumped from the left side to the right side of the dot. Then we have $\xi=1 / 2$.

As observables, $Q^{L}$ and $Q^{R}$ must be gauge invariant, so they must be some functions of the geometric phases $\hat{\phi}^{L}$ and $\hat{\phi}^{R}$. Since the left and right sides are independent, we have $Q=f(\hat{\phi})$ for a given closed loop (here we drop the superscripts for brevity.). To determine the function $f$, we notice that the charge is additive. That is, if we consider a closed loop consisting of two consecutive closed loops $C_{1}$ and $C_{2}$ [see the lower contour in Fig. 2(b)], then we have $Q_{C}=Q_{C_{1}}+Q_{C_{2}}$. On the other hand, the geometric phase is Abelian, implying that $\hat{\phi}_{C}=\hat{\phi}_{C_{1}}+\hat{\phi}_{C_{2}}$. That is, $f\left(\hat{\phi}_{C_{1}}+\hat{\phi}_{C_{2}}\right)=$ $f\left(\hat{\phi}_{C_{1}}\right)+f\left(\hat{\phi}_{C_{2}}\right)$. ¿From this we see that the function must be linear, i.e., $Q=c \hat{\phi}$ with $c$ an undetermined constant. Combining this with the Friedel sum rule which states that charge accumulated inside the scatterer follows $\delta Q_{a}=e /(2 \pi i) \delta \ln (\operatorname{det} S)$, we have $c=-1 /(2 \pi)$. Actually, the argument is applicable to any two arbitrary loops as illustrated in the upper contour in Fig. 2(b). 
In the context of adiabatic pumping, we are interested in pumping cycles without any charge accumulated inside the dot. In this case, we have $\hat{\phi}^{L}=-\hat{\phi}^{R}$. So we may define the circulating current $I$ as $I \equiv Q_{c} / \tau$, which takes the form

$$
I=\frac{\hat{\phi}^{L}-\hat{\phi}^{R}}{4 \pi \tau} .
$$

¿From this we immediately conclude that for an embedded quantum dot with the mirror symmetry, i.e., the left-right symmetry, there is no pumping circulating current. This is consistent with Moskalets and Büttiker [10] who stated that the spatial asymmetry of the scatterer is a necessary condition for the existence of an adiabatic pump effect.

We emphasize that Eq. (3) describes the contribution of a certain discrete energy level to the circulating current. The total current results from summing up all occupied energy levels, which depends on the number of electrons in the ring.

A numerical example. Consider a quantum dot modeled by a potential $V(x)$, which is defined as 0 for $|x| \geq a, V_{1}$ for $-a<x<-b, V_{2}$ for $|x| \leq b$, and $V_{3}$ for $b<x<a$. Here $x$ denotes the coordinate along the ring. The same potential has been used to model the quantum dot embedded in a double path interferometer proposed to directly observe scattering geometric phase [7]. Then the instantaneous spin-independent $2 \times 2$ scattering matrix $S(t)$ for the dot may be determined from the solution of the Schrödinger equation $\left(-\left(\hbar^{2} / 2 m\right) \partial^{2} / \partial x^{2}+V(x)-E\right) \psi=0$. For a dot of size $800 \mathrm{~nm}$ the energy level spacing is of the order of $4.5 \mathrm{meV}$. The Coulomb energy, assuming a dielectric constant of 10 , is of the order of $0.08 \mathrm{meV}$. Thus, the dimension of the dot is such that the Coulomb energy is much less than the separation between the resonances and can be ignored. Also the spin-dependent scattering inside the dot is ignored.

Suppose we periodically and adiabatically vary three gate voltages $V_{1}, V_{2}$, and $V_{3}$. That is, we choose independent external parameters $X^{1}, X^{2}$ and $X^{3}$ as the gate voltages $V_{1}, V_{2}$, and $V_{3}$, respectively, which allows us to control the scatterer in different ways. For instance, we can choose to adiabatically change $V_{1}$ and $V_{2}$ with $V_{3}$ kept constant, i.e., $V_{1}=V_{1}^{0}+\Delta V_{1} \sin \omega t, V_{2}=V_{2}^{0}+\Delta V_{2} \sin \omega t, V_{3}=V_{3}^{0},\left(\Delta V_{1,2} \ll V_{1,2}^{0}\right)$, with $\omega$ being the slow frequency characterizing the adiabaticity.

The allowed discrete $k$ 's may be solved numerically from the dispersion equation, which results in time-dependent discrete energy levels when the dot undergoes an adiabatic cycle. Suppose there are totally $N_{e}=2 M$ electrons in the ring. Then at low temperature, they occupy the lowest $M$ level, with two electrons in each level. The total current $\mathcal{I}$ takes $\mathcal{I} \equiv 2 \sum_{l=1}^{M} I_{(l)}=1 /(2 \pi \tau) \sum_{l=1}^{M}\left(\hat{\phi}_{(l)}^{L}-\hat{\phi}_{(l)}^{R}\right)$, with the subscript $l$ labels the lowest $M$ energy levels. In Fig. 3(a), we plot the total current, $\mathcal{I} \pi \tau$ defined positive clockwise, for 58 electrons as a function of the parameter $\Delta V_{2}$. The current is most sensitive to this parameter. We also plot the contribution to the current from the time dependence of $k$. This contribution is quite small. In Fig. 3(b) we keep the number of electrons constant at 10 and change the size of the ring. When the size of the ring is large compared to the size of the QD ( $\mathrm{L}=100$ and 200 compared to a QD size of 18), then, when increasing the size the current increases only very little. The most interesting behaviour is shown in Fig. 3(c). Here we plot the total current as a 
(a)

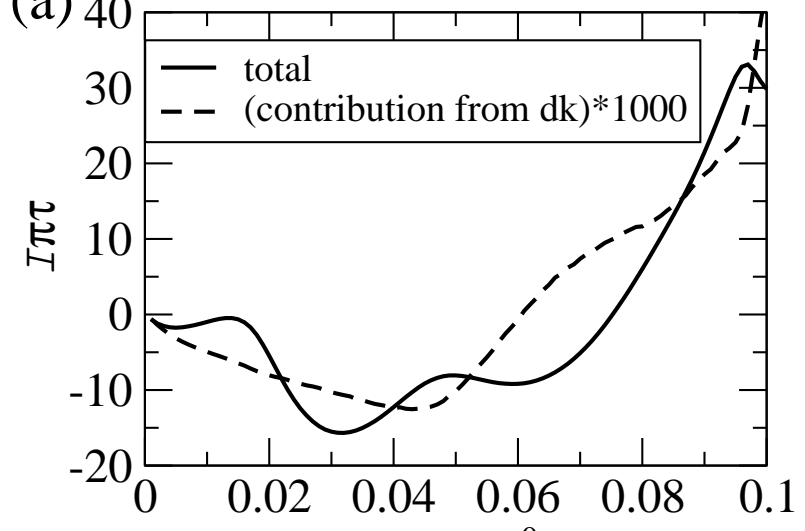

(c) $\Delta \mathrm{V}_{2} / \mathrm{V}_{2}^{0}$ (b)

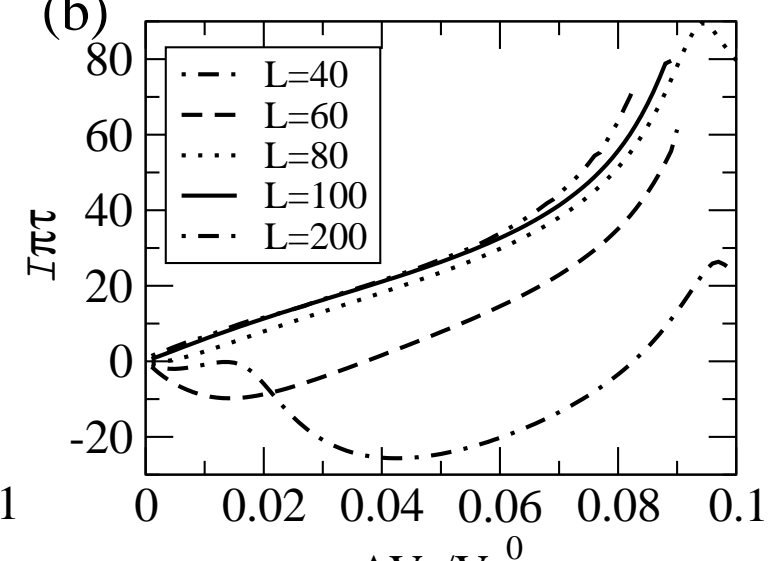

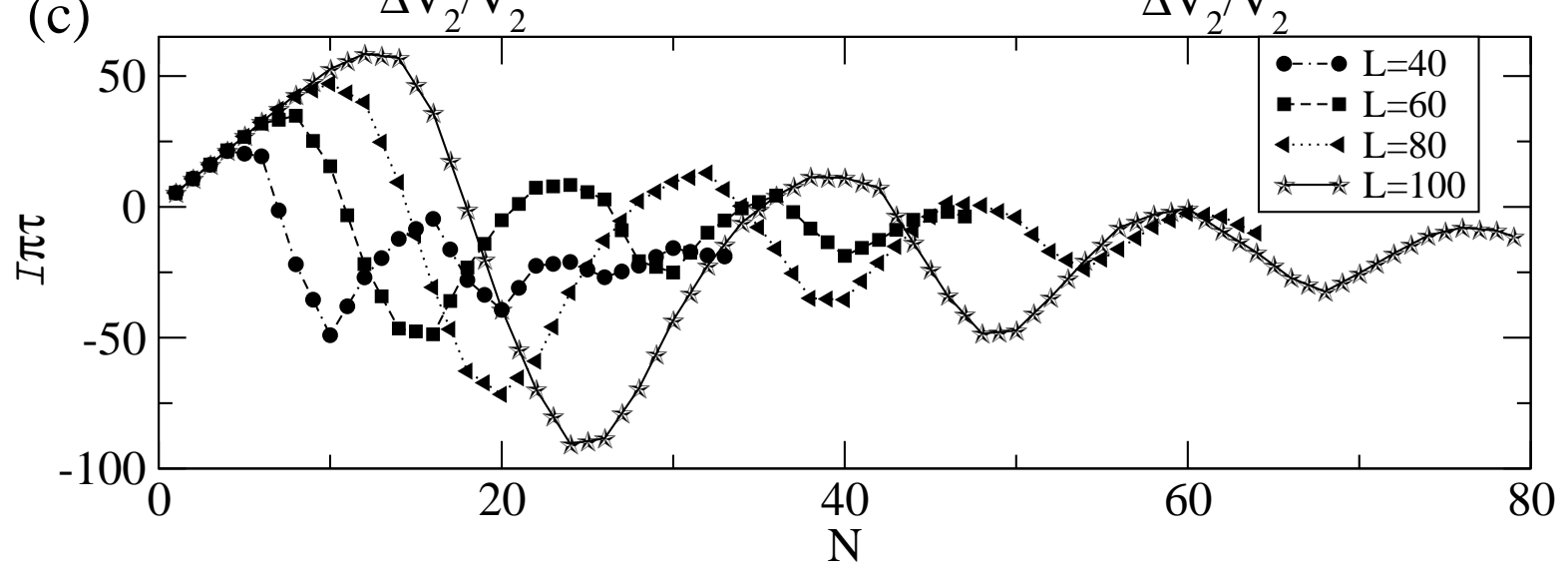

Figure 3. (a) shows the total current as a function of the contour $\Delta V_{2} / V_{2}^{0}$, and contribution to the total current from $\mathrm{d} k$. As we can see the contribution from the time dependence of $k$ is quite small. The ring size is 40 (if the size of the QD is 18 in the same arbitrary units), and the number of electrons in the ring is 58 .

(b) shows the total current for the first 10 electrons for different sizes of the ring as a function of the size of the contour $\Delta V_{2} / V_{2}^{0}$. The current increases as a function of the ring-size for a fixed number of electrons.

(c) shows the total current as a function of the number of electrons in the ring, for different sizes of the ring. Oscillations in the total current is clearly seen. We used $\Delta V_{2}=0.1 V_{2}^{0}$, and $\Delta V_{1}=0.1 V_{1}^{0}$. The size of the $\mathrm{QD}$ is 18 .

function of the number of electrons for different ring sizes. Compared to the persistent current[17] in the ring, there is no large odd/even effect where the current changes sign with the parity. However, in this figure we can see that the current can change sign (thus direction) as a function of the filling, and the effect is larger for a smaller number of electrons. This can be detected by measuring the magnetic field produced by electrons traveling around the ring.

In conclusion, we have studied adiabatic scattering in a ring geometry and established the connection between the circulating (pumping) current and the geometric phases acquired by the time-reversed scattering states based on a pure geometric argument. This may be viewed as a generalization of persistent currents in a mesoscopic 
ring, in the sense that the Aharonov-Bohm effect is a special case of Berry's phases. We have also performed numerical calculations on a quantum dot embedded in a small metallic ring.

\subsection{Acknowledgments}

This work was supported by the Australian Research Council. We thank R.H. McKenzie for helpful discussions and supporting this work.

\section{References}

[1] Berry M V 1984 Proc. Roy. Soc. London, Ser. A 39245

[2] Shapere A and Wilczek F 1989 Geometric Phases in Physics (Singapore: World Scientific)

[3] Jones J A, Vedral V, Ekert A and Castagnoli G 2000 Nature 403 869; Falci G, Fazio R, Palma G, Siewert J and Vedral V 2000 ibid 407 355; Filipp S and Sjöqvist E 2003 Phys. Rev. Lett. 90 050403; Sjöqvist E, et al. 2000 ibid 85 2845; Manini N and Pistolesi F 2000 ibid 853067

[4] Zhou H-Q, Cho S Y, and McKenzie R H 2003 Phys. Rev. Lett. 91186803

[5] Brouwer P W 1998 Phys. Rev. B 5810135

[6] Moskalets M and Büttiker M 2002 Phys. Rev. B 66 035306; Zhou F, Spivak B and Altshuler B 1999 Phys. Rev. Lett. 82 608; Andreev A and Kamenev A 2000 ibid. 85 1294; Avron J E, Elgart A, Graf G M and Sadun L 2001 ibid. 87 236601; Makhlin Y and Mirlin A D 2001 ibid. 87276803

[7] Zhou H-Q, Lundin U, Cho S Y and McKenzie R H 2004 Phys. Rev. B 69113308

[8] Switkes M, Marcus C M, Campman K and Gossard A C 1999 Science 2831905

[9] Cohen D 2003 Phys. Rev. B 68201303

[10] Moskalets M and Büttiker M 2003 Phys. Rev. B 68161311

[11] Landauer R 1957 IBM J. Res. Dev. 1223

[12] Büttiker M, Thomas H and Prètre A 1994 Z. Phys. B 94133

[13] Mailly D, Chapelier C and Benoit A 1993 Phys. Rev. Lett. 702020

[14] Since the adiabatic scatterings in the dot preserves the necessary condition between the characteristic time scales, i.e., $\tau_{d} \ll \tau_{s} \ll \tau$, the dwell time for scattering events can be defined by $\tau_{d}=N / J$, where $J$ is the incident flux, $J=\hbar k / m$, and the number of particles in the dot is given by $N=\int_{\operatorname{dot}}\left|\psi_{\operatorname{dot}}(x)\right|^{2} d x$. Here, $\psi_{\operatorname{dot}}(x)$ is the wave function describing the dot region. In our model of ring geometry, the dwell time is dependent of the discrete $k$ 's due to the boundary condition.

[15] Narnhofer H and Thirring W 1982 Phys. Rev. A 26 3646; Avron J E, Elgart A, Graf G M and Sadun L 2002 J. Math. Phys. 433415

[16] Simon B 1983 Phys. Rev. Lett. 51 2167; Aharonov Y and Anandan J 1987 ibid. 58 1593; Samuel J and Bhandari R 1988 ibid. 602339

[17] Cheung H -F, Gefen Y, Riedel E K and Shih W -H Phys. Rev. B 376050 (1988) 